\title{
Variação na altura e na abundância de colmos e estruturas reprodutivas em populações pioneiras e maduras de Spartina alterniflora Loisel. e S. densiflora Brongn. (Poaceae) do sul do Brasil
}

\author{
Vera Lúcia Caetano', Gabriela Greis², Adriana Martins Guedes de Azevedo³ \\ \& César Serra Bonifácio Costa ${ }^{4}$
}

\author{
' Faculdade Cenecista de Osório, Curso de Ciências Biológicas, Rua 24 de Maio, 141 Centro, CEP 95520-000, Osório, RS. \\ 2 Universidade Federal do Rio Grande, Instituto de Oceanografia, Programa de Pós-Graduação em Oceanografia Biológica, Av. Itália, \\ km 8, Bairro Carreiros, CEP 96203-900, Rio Grande, RS. \\ ${ }^{3}$ Universidade Federal do Rio Grande, Instituto de Oceanografia, Programa de Pós-Graduação em Aquicultura. \\ Av. Itália, km 8, Bairro Carreiros, CEP 96203-900, Rio Grande, RS. \\ ${ }^{4}$ Universidade Federal do Rio Grande, Instituto de Oceanografia, Laboratório de \\ Biotecnologia de Halófitas Av. Itália, km 8, Bairro Carreiros, CEP 96203-900, Rio Grande, RS. docosta@furg.br
}

Recebido em 27.IX.2017

Aceito em 03.VIII.2017

DOI $10.21826 / 2446-8231201772209$

RESUMO - Este estudo visou quantificar e interpretar a variabilidade espacial na altura das plantas e na abundância de colmos e de estruturas reprodutivas das gramíneas dominantes de marismas Spartina alterniflora Loisel. e Spartina densiflora Brongn. em populações pioneiras ( $<10$ anos de colonização) e maduras ( $>50$ anos) no sul do Brasil. Para ambas as espécies, os colmos foram mais altos em populações pioneiras ( $p<0,01)$. A população pioneira de $S$. densiflora investe massivamente na densidade dos colmos $\left(10.084\right.$ colmos $\left.\mathrm{m}^{-2}\right)$, produz mais inflorescências $\left(557 \mathrm{~m}^{-2}\right)$ e cariopses $\left(16.258 \mathrm{~m}^{-2}\right)$ do que a população madura (131 inflorescências $\mathrm{m}^{-2}$ e 2.199 cariopses $\left.\mathrm{m}^{-2}\right)$. Maiores valores de redox e menos fósforo disponível no sedimento com o afastamento da linha d'água parecem explicar menores densidades de colmos e inflorescência dentro da população madura de $S$. densiflora. Na população pioneira de $S$. alterniflora, $14 \%$ dos colmos floresceram e produziram apenas 0,4 cariopses por inflorescência e 76 cariopses $\mathrm{m}^{-2}$, enquanto a população madura não floresceu.

Palavras-chave: densidade, fecundidade, gradientes ambientais, ocupação espacial

ABSTRACT - Variation of the tiller height and abundance of tillers and reproductive structures in pioneer and mature populations of Spartina alterniflora Loisel. and $\boldsymbol{S}$. densiflora Brongn. (Poaceae) in southern Brazil. This study aimed to quantify and elucidate the spatial variability of plant height and abundance of tillers and reproductive structures of the salt marsh grasses Spartina alterniflora Loisel. and Spartina densiflora Brongn. within and between pioneer $(<10$ years of colonization) and mature stands ( $>50$ years of colonization) in southern Brazil. Both pioneer populations had taller tillers $(\mathrm{p}<0.01)$ than mature populations. Pioneer populations of $S$. densiflora also showed massive investment on tiller density $\left(10,084 \mathrm{~m}^{-2}\right)$, more inflorescences $\left(557 \mathrm{~m}^{-2}\right)$ and higher fecundity $\left(16,258\right.$ seeds $\left.\mathrm{m}^{-2}\right)$ than the mature population $\left(131\right.$ inflorescence $\mathrm{m}^{-2} ; 2,199 \mathrm{seeds}$ $\mathrm{m}^{-2}$ ). High redox and reduction of phosphorus availability in the sediment away from the water line seem to explain decreasing densities of tiller and inflorescences inside $S$. densiflora mature populations. Fourteen percent of pioneer S. alterniflora tillers flowered and, on average, produced only 0.4 seeds per inflorescence and 76 seeds $\mathrm{m}^{-2}$, whereas mature population did not flower.

Keywords: density, environmental gradients, fecundity, spatial occupation

\section{INTRODUÇÃO}

Marismas são ambientes entremarés com sedimento inconsolidado recoberto por vegetação vascular herbácea. Elas cumprem funções vitais como sequestro de carbono, que pode ser acumulado por sua cobertura vegetal nas estruturas orgânicas dos caules, raízes, rizomas e detrito depositado na superfície e solo da zona entremarés (Martinetto et al. 2016), sustentação trófica dos ecossistemas costeiros, estabilização da costa e elo dos ciclos biogeoquímicos de vários elementos (Costa et al. 1997, Peixoto \& Costa 2004, Cunha et al. 2005, Costa et al. 2009). Poucas espécies de plantas colonizam as marismas devido a grande hidrodinâmica e estresse hídrico, sendo comuns grandes extensões com formações vegetais monoespecíficas.

As gramíneas do gênero Spartina Schreb. (Chloridoideae, Poaceae) são halófitas (tolerantes a altas salinidades) representativas em ambientes costeiros e dominantes de marismas em várias partes do mundo (Proffit et al. 2003, Nieva et al. 2005, Chung 2006). Suas espécies são reconhecidas como plantas bioengenheiras, por sua capacidade de colonizar áreas desnudas alagadas periodicamente pelo mar, reduzir a hidrodinâmica e promover a deposição do sedimento com sua densa copa, 
além de fixar o sedimento depositado com seu sistema radicular (Hulzen et al. 2007, Mendonça \& Costa 2008, Marangoni \& Costa 2012).

Várias espécies de Spartina apresentam uma grande variabilidade da altura e abundância de seus colmos, frequentemente atribuída a respostas fenotípicas a fatores ambientais, tais como disponibilidade de nutrientes (Linthurst \& Seneca 1981, Castellanos et al. 1998, Tyler \& Zieman 1999, Azevedo et al. 2016), salinidade ou tempo de inundação-potencial redox (Nieva et al. 2001, Castillo et al. 2005, Hulzen et al. 2007). As formas de crescimento destas plantas também influenciam sua ação bioengenheira no desenvolvimento da marisma. Por exemplo, plantas de populações de Spartina com colmos mais altos e maiores densidades de colmos exercem maior impacto de redução das condições hidrodinâmicas, possibilitando a deposição de sedimento fino e o enriquecimento de nutrientes (Tyler \& Zieman 1999, Seliskar et al. 2002). Visto que a propagação vegetativa (crescimento clonal) é, frequentemente, a forma mais importante de colonização e manutenção de populações do gênero Spartina (Thompson et al. 1991, Castellanos et al. 1998, Tyler \& Zieman 1999, Costa et al. 2003, Marangoni \& Costa 2012), o estímulo ou inibição do desenvolvimento em altura e da densidade dos colmos por fatores locais têm impacto direto sobre a adaptabilidade das plantas (Lovett Doust \& Lovett Doust 1982). Maiores quantidades de nutrientes aceleram a perfilhação e o crescimento vertical das plantas e logo sua capacidade de modificar seu próprio hábitat (Bertness 1991, Castillo et al. 2005). As moitas destas plantas tendem a crescer centrifugamente colonizando planos entremarés (Kittelson \& Boyd 1997, Marangoni \& Costa 2012), tornando-se mais altas no centro (Castellanos et al. 1998, Hulzen et al. 2007, Mendonça \& Costa 2008). Consequentemente, ao longo da formação de uma marisma de Spartina, as características do crescimento vegetativo podem se alterar.

A biologia reprodutiva das espécies de Spartina ainda é pouco conhecida (Fang et al. 2004). A densidade total de colmos de Spartina densiflora Brongn. relaciona-se diretamente com a produção de colmos reprodutivos e de sementes em suas populações (Nieva et al. 2001), enquanto que Spartina alterniflora Loisel. (Dai \& Wiegert 1996), S. maritima (M.A. Curtis) Fernald (Castellanos et al. 1998) e S. anglica C.E. Hubbard (Thompson et al. 1990, 1991) apresentam uma relação inversa entre densidade populacional e porcentagem de indivíduos com inflorescência. Algumas espécies muito estudadas, como S. alterniflora, produzem uma grande quantidade de pólen com alta fertilidade (Daehler \& Strong 1994), entretanto o número de sementes viáveis produzidas é muito errático e os fatores causais deste fenômeno ainda não foram esclarecidos (Callaway \& Josselyn 1992, Daehler 1998). S. densiflora também apresenta grande variabilidade na formação de colmos reprodutivos (Nieva et al. 2001, Vicari et al. 2002), mas pouco é conhecido o seu potencial reprodutivo, particularmente sua produção de sementes.
No sudoeste do Oceano Atlântico, S. alterniflora e $S$. densiflora ocorrem simpatricamente em marismas (Costa \& Davy 1992, Costa et al. 2003, Marangoni \& Costa 2012), sendo importantes espécies pioneiras e estruturadoras destes ambientes. Amplamente distribuída na costa atlântica das Américas, no estuário da Lagoa dos Patos (RS), $S$. alterniflora domina pisos entremarés baixos, formando coberturas em áreas euhalinas e mesohalinas, alagadas 50\% do tempo (Costa 1998). Spartina densiflora ocorre na costa atlântica da América do Sul do estado de São Paulo até o sul da Argentina (Costa et al. 2003, Martinetto et al. 2016), sendo espécie invasora na costa pacífica da América do Norte, Europa e África (Kittelson \& Boyd 1997, Nieva et al. 2001, 2005). Spartina densiflora é uma das plantas de maior amplitude vertical na zona entremarés do estuário da Lagoa dos Patos, ocorrendo como pioneira na colonização das marismas baixas, mas dominando os pisos entremarés intermediários, sujeitos a alagamento esporádico (cerca de $20 \%$ do ano) (Costa 1998). Pode estar acompanhada por S. alterniflora ou outras halófitas (Scirpus maritimus, Sarcocornia ambigua) ou mesmo espécies transicionais de outros hábitats (Costa et al. 2003, Peixoto \& Costa 2004).

De forma a compreender os fatores que determinam a variação espacial na altura de colmos e na produção de estruturas reprodutivas de $S$. alterniflora e $S$. densiflora em marismas no sul do Brasil, populações pioneiras $(<10$ anos de colonização) e maduras ( $>50$ anos) destas espécies tiveram suas características biométricas quantificadas ao longo do gradiente de elevação local.

\section{MATERIAL E MÉTODOS}

O estudo foi realizado na Ilha da Pólvora, localizada no estuário da Lagoa dos Patos, no município de Rio Grande (RS; $32^{\circ} 01^{\prime} \mathrm{S}, 52^{\circ} 06^{\prime} \mathrm{W}$ ) (Fig. 1). O estuário da Lagoa dos Patos apresenta um regime micromareal (amplitudes máximas de marés astronômicas de $0,5 \mathrm{~m}$ ), com um forte componente meteorológico que determina o alagamento irregular das marismas por águas de salinidade variável (Costa 1998, Costa et al. 2003, Marangoni \& Costa 2012). O clima local é temperado quente com expressivas flutuações sazonais de fotoperíodo, radiação solar, pluviosidade e temperatura. Os meses de menores e maiores temperaturas médias são, respectivamente, julho $\left(12,7^{\circ} \mathrm{C}\right)$ e janeiro $\left(24,6^{\circ} \mathrm{C}\right)$ (Vieira 1983$)$.

No outono de 2005 , duas populações de S. alterniflora e outras duas de $S$. densiflora, com distintas idades de colonização da zona entremarés foram amostradas quanto a parâmetros biométricos dos colmos e estruturas reprodutivas. Durante o outono estas duas espécies atingem os seus picos de crescimento vegetativo e reprodutivo no sul do Brasil (Giacobbo \& Boechat 1988, Costa et al. 2003, Peixoto \& Costa 2004, Cunha et al. 2005), desta maneira constitui o melhor período para avaliar seus máximos desempenhos biológicos nos diferentes habitats das marismas. 


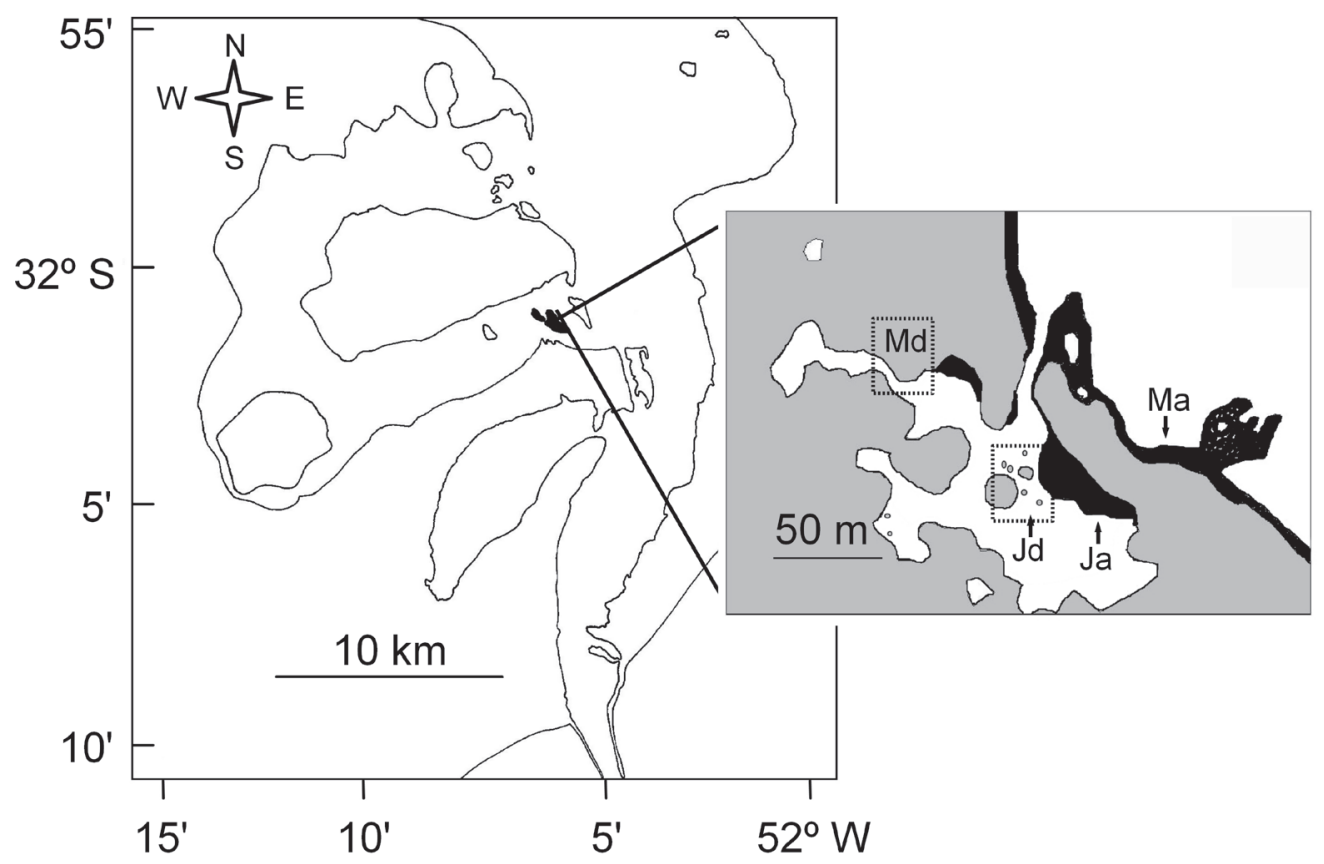

Fig. 1. Localização da Ilha da Pólvora no estuário da Lagoa dos Patos, município de Rio Grande, RS. Detalhe da distribuição das marismas dominadas pelas coberturas de $S$. alterniflora (preto) e $S$. densiflora (cinza). As populações jovem e madura de S. alterniflora foram identificadas pelos códigos ja e Ma; as de $S$. densiflora pelos códigos jd e Md.

Foram amostradas populações pioneiras e maduras de ambas as espécies que possuíam, respectivamente, menos de 10 anos e mais de 50 anos de ocupação das áreas entremarés, conforme interpretação de fotografias aéreas obtidas entre 1947 e 2003. Utilizou-se para processamento das fotos digitalizadas e análise espacial o programa SIG SPRING 4.3, sendo a metodologia detalhada em Marangoni \& Costa (2012). A localização geográfica de cada população na ilha foi realizada com a ajuda de um GPS eTrex Garmin. A altura topográfica de cada marisma foi relacionada com o nível médio da Lagoa para a década 1995-2005 (NML), através de medidas simultâneas do nível de água na área amostral da marisma e no ponto de monitoramento hidrológico do programa de Pesquisa Ecológica de Longa Duração-PELD, localizado aproximadamente a $1,5 \mathrm{~km}$ de distância. A curva da frequência de alagamento em diferentes níveis do piso entremarés do período 19952005 demonstrou um ótimo ajuste a um polinômio de terceira ordem $\left(r^{2}=0,98\right)$, e esta equação foi utilizada para calcular a frequência de alagamento para a altura média das áreas ocupadas por populações pioneiras e maduras de ambas as espécies. Em abril/2005, foi coletada uma amostra de sedimento na região central de cada população das duas espécies, através de um tubo de PVC com $10 \mathrm{~cm}$ de diâmetro e $50 \mathrm{~cm}$ de comprimento. Após a coleta de uma coluna sedimentar com cerca de $40 \mathrm{~cm}$, o potencial de oxirredução (redox) foi medido diretamente no sedimento nas profundidades $0,5,10$ e $15 \mathrm{~cm}$, através da inserção de um sensor de platina "heavy dust" conectado a um medidor da marca DigiSense.

Em cada população de $S$. alterniflora foram amostrados 15 quadrados de $0,25 \mathrm{~m}^{2}$, equidistantes $2 \mathrm{~m}$, ao longo de um de transecção a partir do plano de lama, na borda inferior da área vegetada de cada população, até o interior da área vegetada. Este tipo de amostragem permite avaliar o efeito do afastamento do plano de lama (próximo do corpo de água adjacente) sobre o desempenho das plantas (i.e., componente intrapopulacional da variabilidade morfológica, Proffit et al. 2003; Hulzen et al. 2007).

Populações de $S$. densiflora também foram amostradas com 15 quadrados cada, entretanto com uma área amostral de $0,0625 \mathrm{~m}^{2}$ devido a grande densidade de colmos. A colonização de planos de lama por $S$. densiflora ocorre a partir de um crescimento vegetativo radial de plântulas ou fragmentos de rizomas depositados pela maré, formando manchas vegetadas na forma de círculos. Em suas análises da ocupação espacial das marismas do estuário da Lagoa dos Patos por plantas de S. densiflora, Costa \& Marangoni (1997) e Marangoni \& Costa (2012) determinaram taxas médias de expansão radial dos tufos de colmos desta espécie entre 5 e $13 \mathrm{~cm}^{2}{ }^{-1}$. Assim, para amostragem da população pioneira ( $<10$ anos de colonização) foram selecionados círculos vegetados isolados no plano de lama com $0,5 \mathrm{~m}$ a 1,0m de diâmetro, sendo examinado um quadrado na área central de cada círculo. Já, populações maduras ( $>50$ anos) foram caracterizadas por círculos vegetados com diâmetro acima de $60 \mathrm{~m}$ e os quadrados amostrais tiveram a mesma disposição espacial utilizada para S. alterniflora.

Para ambas as espécies, a amostragem consistiu no registro em cada quadrado amostral do número de colmos vivos (com folhas verdes), da altura dos maiores colmos (10 maiores para $S$. densiflora e apenas o maior de todos para S. alterniflora) e do número de colmos reprodutivos (com inflorescências). Foi calculada também a porcentagem 
de colmos reprodutivos pelo número total de colmos vivos registrados por quadrado amostral. Adicionalmente, para ambas as populações das duas espécies, foram coletadas 15 inflorescências de forma aleatória em área adjacente aos quadrados quantificados, sendo estimados em cada inflorescência os números de racemos (ramos secundários da inflorescência), de espiguetas (um par de glumas estéreis e um antécio; unidades reprodutivas) e de cariopses (sementes potencialmente viáveis). A fecundidade foi estimada multiplicando-se o número médio de cariopses pelo número médio de colmos reprodutivos por unidade de área da marisma.

Para todas variáveis quantificadas foram testados os pressupostos (Zar 2010) de normalidade (teste de Kolmogorov-Smirnov) e a homocedasticidade (teste de Bartlett) a 5\% de significância. De forma a atender estes requisitos para análises estatísticas paramétricas, algumas variáveis tiveram que ser transformadas. As variáveis densidade total de colmos, densidade de colmos reprodutivos e porcentagem de colmos reprodutivos foram transformados, respectivamente, pelas funções $\log _{10} \mathrm{x}, \log _{10}(\mathrm{x}+1)$ e $\operatorname{ArcSen}(\sqrt{\mathrm{x}}+0,5)$. Para cada espécie, as variáveis biométricas dos colmos, a abundância de estruturas reprodutivas nas inflorescências e a fecundidade foram comparadas entre populações através de Análises de Variância (ANOVA) unifatoriais, seguidas do teste de post-hoc de Tukey, considerando 5\% de significância (Zar 2010). As percentagens da variação total destas variáveis contabilizadas pelos efeitos interpopulacional e intrapopulacional foram estimadas, respectivamente, pelos valores da soma dos quadrados da variação explicada (entre populações) e da soma dos quadrados do resíduo, ambos divididos pelo total da soma dos quadrados (Proffit et al. 2003, Zar 2010). Adicionalmente, de forma a auxiliar na interpretação da variabilidade espacial, para as variáveis que demonstraram mais de $50 \%$ de sua variabilidade vinculada ao efeito intrapopulacional, foram efetuadas regressões lineares entre seus valores e à distância de cada quadrado amostral até a linha d'água, i.e., o plano de lama adjacente (Hulzen et al. 2007).

\section{RESULTADOS}

As populações pioneiras de $S$. alterniflora e $S$. densiflora ocupavam planos entremarés entre -15 e -9 cm NML (nível médio da lagoa estimado entre 1995-2005), que foram em média alagados cerca de $72 \%$ do tempo ao longo da década. A população madura de $S$. densiflora encontrava-se entre $+2 \mathrm{e}+19 \mathrm{~cm}$ NML (média \pm desvio padrão $=+7 \pm 2 \mathrm{~cm}$ NML; alagada $40 \pm 3 \%$ do tempo), localizada acima no entremarés da população madura de $S$. alterniflora (entre $-1 \mathrm{e}+8 \mathrm{~cm}$ NML; $+4 \pm 1 \mathrm{~cm} \mathrm{NML}$; alagada $45 \pm 2 \%$ do tempo). Baixos potenciais redox entre $-15 \mathrm{e}-125 \mathrm{mV}$ foram observados entre $0-5 \mathrm{~cm}$ de profundidade no sedimento das populações pioneiras de S. alterniflora e S. densiflora (Fig. 2), mesmo durante os meses de águas baixas do outono

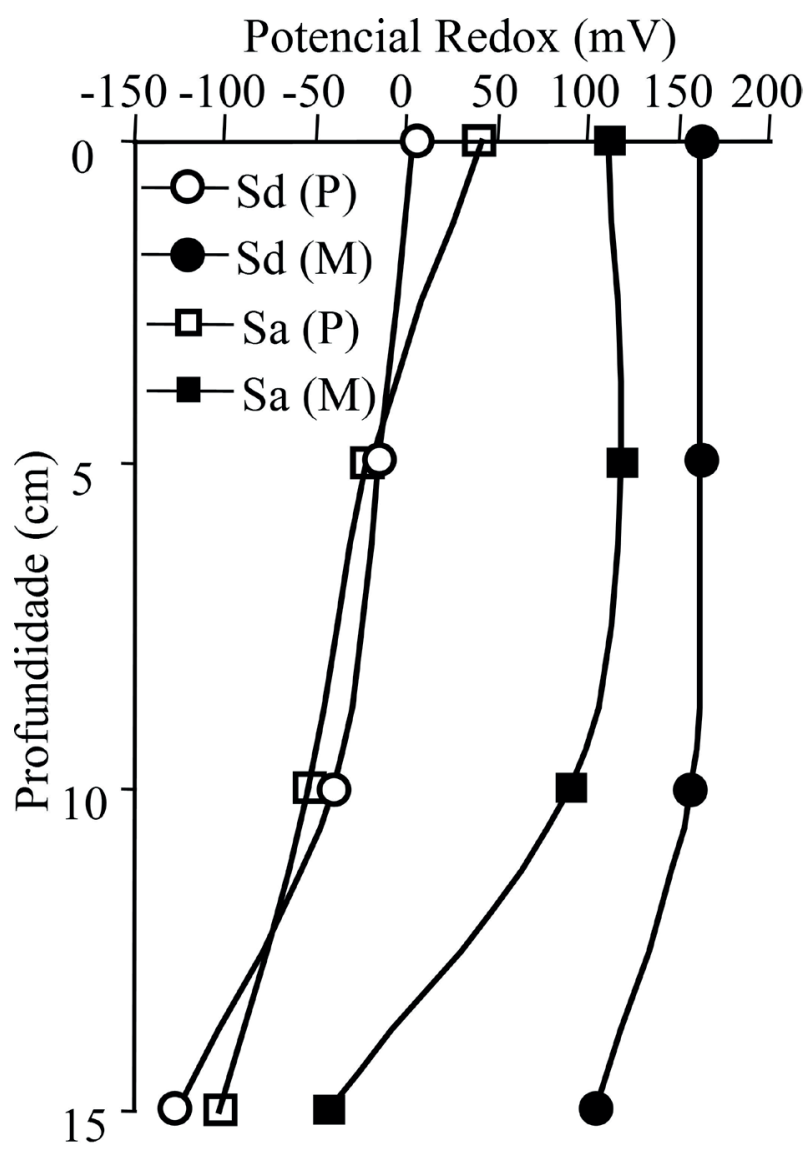

Fig.2. Perfil vertical do potencial redox $(\mathrm{mV})$ no sedimento de populações pioneiras (P) e maduras (M) de S. alterniflora $(\mathrm{Sa})$ e $S$. densiflora $(\mathrm{Sd})$ em marismas da Ilha da Pólvora, Rio Grande (RS).

(Costa et al. 2003). As populações maduras de ambas as espécies, por outro lado, estavam submetidas a ambientes mais oxidados (entre $+89 \mathrm{e}+163 \mathrm{mV}$ ) nos primeiros 15 $\mathrm{cm}$ da camada superficial do sedimento (Fig. 2).

Os colmos da população pioneira de S. alterniflora apresentaram altura média $(79 \pm 10 \mathrm{~cm})$ significativamente $(\mathrm{F}=27,2 ; \mathrm{p}<0,001)$ maior do que os da população madura $(64 \pm 4 \mathrm{~cm})$. A densidade dos colmos vegetativos $(\mathrm{F}=139,1$; $\mathrm{p}<0,001)$ e a densidade total de colmos $(\mathrm{F}=100,4 ; \mathrm{p}<0,001)$ da população madura de $S$. alterniflora foram duas vezes maiores do que as da sua população pioneira (Tab. 1). O investimento na formação de estruturas reprodutivas foi também diferenciado entre as populações amostradas de $S$. alterniflora. Em média 14\% dos colmos da população pioneira apresentaram inflorescências ( $28 \pm 14$ inflorescências $\left.\mathrm{m}^{-2}\right)$, enquanto a população madura não floresceu. A população pioneira produziu em média $126,0 \pm 61,3$ espiguetas por inflorescência (mín. 67, máx. 274) e apenas $0,4 \pm 1,0$ cariopses por inflorescência (Tab. 2). Considerando a densidade de colmos reprodutivos, a fecundidade estimada para esta população foi de apenas 76 cariopses $\mathrm{m}^{-2}$.

A população pioneira de $S$. densiflora apresentou uma média das alturas máximas dos colmos $(121 \pm 12 \mathrm{~cm})$ significativamente maior $(\mathrm{F}=17,9 ; \mathrm{p}<0,001)$ do que da população madura $(103 \pm 11 \mathrm{~cm})$. A densidade de colmos 
Tabela 1. Médias ( \pm desvio-padrão) de altura dos colmos (A), das densidades de colmos vegetativos (V), de colmos reprodutivos (R) e total de colmos (T), bem como da porcentagem de colmos reprodutivos $(\mathrm{Fi} \%)$ nas populações pioneiras e maduras de Spartina alterniflora $(\mathrm{n}=15)$ e $S$. densiflora $(\mathrm{n}=15)$ nas marismas da Ilha da Pólvora $(\mathrm{RS})$. Os resultados das Análises de Variância interpopulacional também são apresentados.

\begin{tabular}{|c|c|c|c|c|}
\hline \multirow{2}{*}{ S. alterniflora } & \multicolumn{2}{|c|}{ Populações } & \multicolumn{2}{|c|}{ Interpopulaconal } \\
\hline & Pioneira & Madura & $\mathrm{F}$ & $\mathrm{p}$ \\
\hline $\mathrm{A}(\mathrm{cm})$ & $79 \pm 10$ & $64 \pm 4$ & 27,2 & $<0,001$ \\
\hline $\mathrm{T}\left(\operatorname{colmos} \mathrm{m}^{-2}\right)$ & $227 \pm 44$ & $453 \pm 76$ & 100,4 & $<0,001$ \\
\hline $\mathrm{V}\left(\operatorname{colmos} \mathrm{m}^{-2}\right)$ & $190 \pm 37$ & $453 \pm 76$ & 158,9 & $<0,001$ \\
\hline $\mathrm{R}\left(\operatorname{colmos} \mathrm{m}^{-2}\right)$ & $28 \pm 14$ & 0 & $1.219,0$ & $<0,001$ \\
\hline $\mathrm{Fi}(\%)$ & $14 \pm 5$ & 0 & 453,3 & $<0,001$ \\
\hline
\end{tabular}

\begin{tabular}{|c|c|c|c|c|}
\hline \multirow{2}{*}{ S. densiflora } & \multicolumn{2}{|c|}{ Populações } & \multicolumn{2}{|c|}{ Interpopulacional } \\
\hline & Pioneira & Madura & $\mathrm{F}$ & $\mathrm{p}$ \\
\hline $\mathrm{A}(\mathrm{cm})$ & $120 \pm 12$ & $103 \pm 11$ & 17,9 & $<0,001$ \\
\hline $\mathrm{T}\left(\operatorname{colmos} \mathrm{m}^{-2}\right)$ & $10.084 \pm 2.138$ & $3.616 \pm 1.383$ & 71,7 & $<0,001$ \\
\hline $\mathrm{V}\left(\operatorname{colmos} \mathrm{m}^{-2}\right)$ & $9.528 \pm 2.078$ & $3.485 \pm 1350$ & 65,8 & $<0,001$ \\
\hline $\mathrm{R}\left(\operatorname{colmos} \mathrm{m}^{-2}\right)$ & $557 \pm 317$ & $131 \pm 107$ & 22,5 & $<0,001$ \\
\hline Fi $(\%)$ & $6 \pm 3$ & $4 \pm 3$ & 3,4 & 0,074 \\
\hline
\end{tabular}

Tabela 2. Número de estruturas reprodutivas produzidas por populações pioneiras e maduras de Spartina alterniflora e $S$. densiflora nas marismas da Ilha da Pólvora (RS). NRAC= número total de racemos por inflorescência; $\mathrm{NESPG}=$ número total de espiguetas por inflorescência; $\mathrm{CAR}=$ número de cariopses por inflorescência; $\mathrm{FEC}=$ fecundidade, número de cariopses por $\mathrm{m}^{-2}$.

\begin{tabular}{lccc}
\hline $\begin{array}{l}\text { Estruturas } \\
\text { Reprodutivas }\end{array}$ & $\begin{array}{c}\text { S. alterniflora } \\
\text { (pioneira) }\end{array}$ & $\begin{array}{c}\text { S. alterniflora } \\
\text { (madura) }\end{array}$ & $\begin{array}{c}\text { S. densiflora } \\
\text { (pioneira) }\end{array}$ \\
\hline NRAC & $7,1 \pm 2,8$ & 0 & $\begin{array}{c}\text { S. densiflora } \\
\text { (madura) }\end{array}$ \\
NESPG & $126,0 \pm 61,3$ & 0 & $4,8 \pm 3,4$ \\
CAR & $0,4 \pm 1,0$ & 0 & $198,8 \pm 68,3$ \\
FEC & 76 & 0 & $29,2 \pm 31,5$ \\
\hline
\end{tabular}

vegetativos da população pioneira de $S$. densiflora foi 9.528 \pm 2.078 colmos $\mathrm{m}^{-2}$, cerca de três vezes maior $(\mathrm{F}=65,8$; $\mathrm{p}<0,001)$ do que a da população madura $(3.485 \pm 1350$ colmos $\left.\mathrm{m}^{-2}\right)$ (Tab. 1). Adicionalmente, a população pioneira formou quatro vezes mais inflorescências do que a população madura $(\mathrm{F}=22,5 ; \mathrm{p}<0,001)$, respectivamente, $557 \pm 317$ e $131 \pm 107$ inflorescências $\mathrm{m}^{-2}$, respectivamente. Apesar da porcentagem média de colmos com inflorescências nas populações pioneira e madura não ter apresentado diferença estatística, variando de $6 \pm 3 \%$ a $4 \pm 3 \%$, (Tab. 1 ), a produção das estruturas reprodutivas de $S$. densiflora demonstrou um investimento nitidamente diferenciado entre estas populações. A população pioneira de $S$. densiflora produziu duas vezes mais racemos $(9,8 \pm 3,4$ racemos inflorescência $\left.{ }^{-1}\right)$ e espiguetas por inflorescências $(198,8$ $\pm 68,3$ espiguetas inflorescência $\left.{ }^{-1}\right)$ do que a população madura $\left(4,8 \pm 1,7\right.$ racemos inflorescência ${ }^{-1} ; 94 \pm 29,1$ espiguetas inflorescência ${ }^{-1}$ ) (Tab. 2). Entretanto, o número de cariopses produzidas por inflorescências foi apenas $42 \%$ maior na população pioneira $(29,2 \pm 31,5$ cariopses inflorescência $\left.{ }^{-1}\right)$ do que na população madura $(20,5 \pm$ 10,2 cariopses inflorescência ${ }^{-1}$ ). Apesar da maior eficácia reprodutiva da população madura (i.e., cariopses produzidas por espiguetas), a grande densidade de colmos e a proporção de colmos que produzem inflorescências da população pioneira faz com que sua fecundidade seja cerca de 8 vezes maior (16.258 cariopses $\mathrm{m}^{-2}$ ) do que a da população madura (2.199 cariopses $\left.\mathrm{m}^{-2}\right)$ (Tab. 2).
A decomposição da variância mostrou que, para ambas as espécies, a altura dos colmos foi igualmente explicada por diferenças entre estágios de desenvolvimento das marismas e por variação espacial dentro das populações (Figs. 3A, B), enquanto a densidade total e de colmos vegetativos eram 70$80 \%$ contabilizada por diferenças entre populações (Fig. 3C). A abundância dos colmos reprodutivos (densidade e porcentagem na população) comportou-se de forma bem diferente entre as espécies. Para S. alterniflora a abundância das estruturas reprodutivas é contabilizada em mais de $90 \%$ por diferenças entre estágios de desenvolvimento das marismas, enquanto para $S$. densiflora $50-90 \%$ destas estruturas é explicada por variação dentro das populações (Figs. 3D, E).

Quanto as variáveis fortemente influenciadas pela variação espacial, as alturas médias dos colmos de $S$. densiflora da marisma madura não se relacionaram significativamente $\left(r^{2}=0,02 ; p=0,64\right)$ com o afastamento da linha d'água (do plano de lama adjacente) (Fig. 4A). Entretanto, as alturas médias dos colmos dentro das populações pioneira e madura de S. alterniflora aumentaram significativamente $(p<0,05)$ com o afastamento da linha d'água (Figs. 4B, C). Adicionalmente, a densidade e a percentagem de colmos reprodutivos de $S$. densiflora na marisma madura reduziram significativamente com o aumento da distância da linha d'água (Figs. 4C, D), que explicou de 26 a $56 \%$ da variabilidade destas duas variáveis dentro das populações. 


\section{DISCUSSÃO}

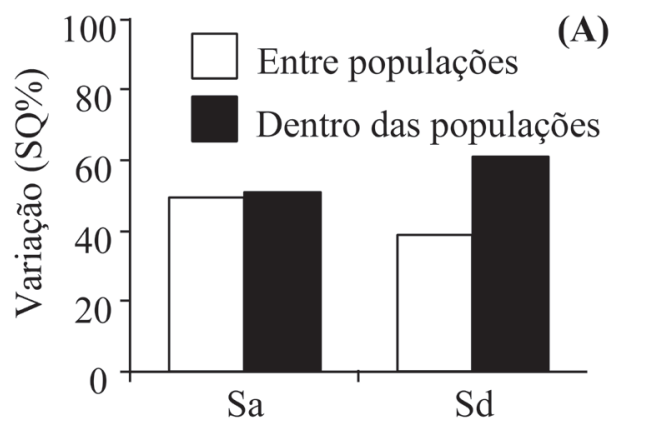

胥
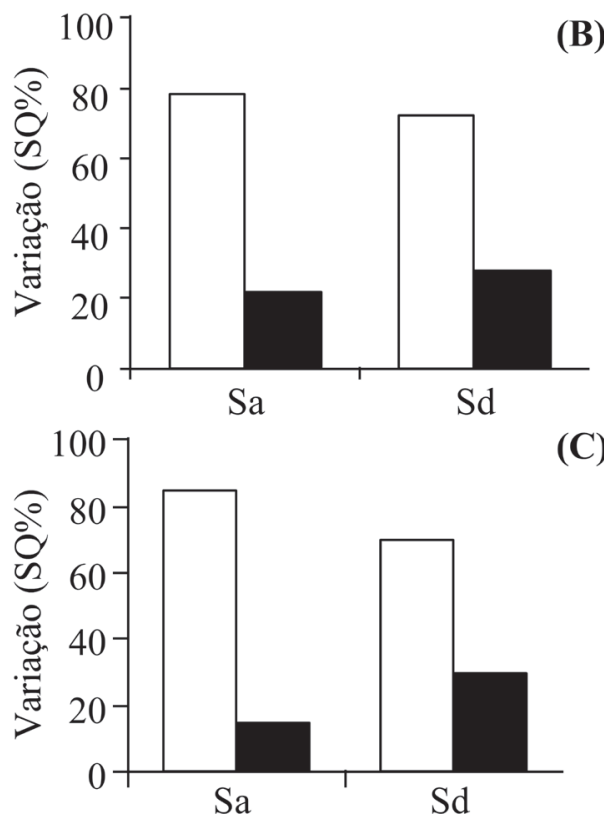

(C)
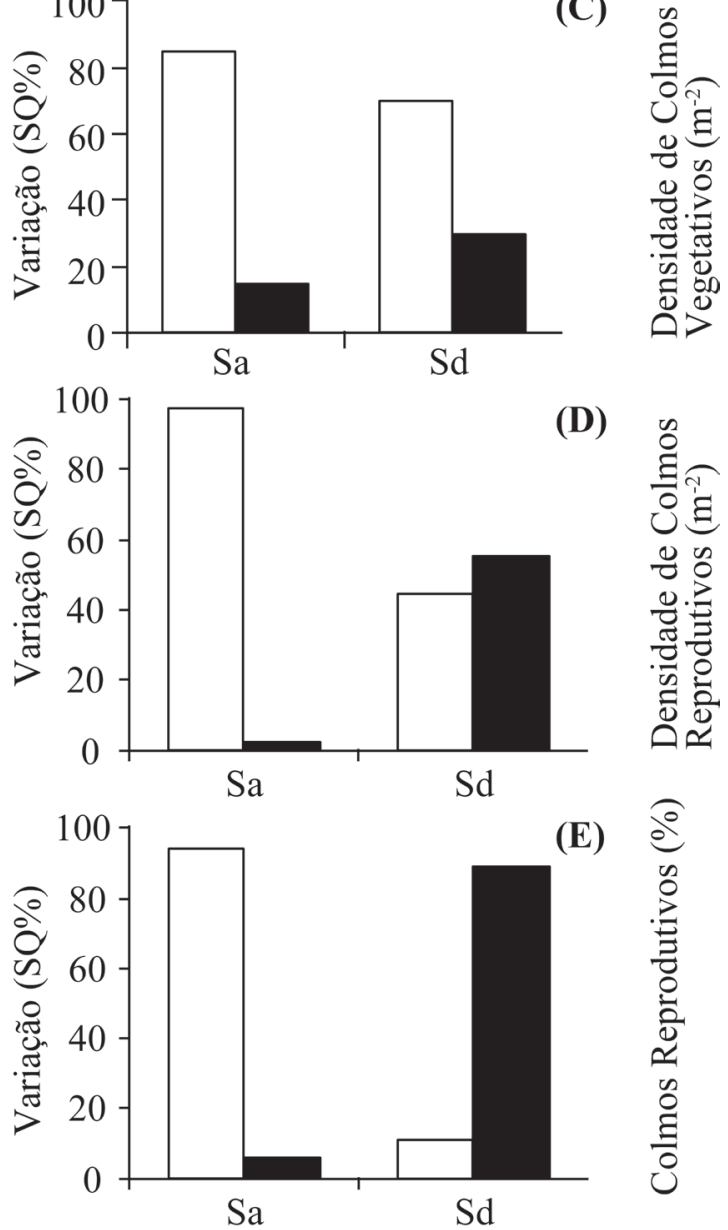

Figs.3 A-E. Variações nas alturas (A) e densidades de colmos (totais, vegetativos e reprodutivos) (B-D), como da percentagem de colmos reprodutivos (E) contabilizadas por Análises de Variância entre e dentro das populações pioneiras e maduras de $S$. alterniflora $(\mathrm{Sa})$ e $S$. densiflora $(\mathrm{Sd})$ em marismas da Ilha da Pólvora, Rio Grande (RS). SQ\% = percentagem do total da soma dos quadrados.
As duas espécies de Spartina demonstraram marcada variabilidade morfológica e distintos padrões de investimento reprodutivo entre populações pioneiras e maduras. Adicionalmente, características da forma de crescimento como a altura e a densidade dos colmos demonstraram uma grande variabilidade espacial (intrapopulacional) relacionada com a distância da borda plano de lama-marisma.

As populações maduras encontravam-se em pisos entremarés mais altos do que as populações pioneiras, diferença resultante do soerguimento biogênico da marisma, que induz distintas condições de alagamento capazes de influenciar o crescimento e a reprodução das plantas. Os colmos das populações maduras de ambas as espécies apresentaram menores alturas do que os das populações pioneiras. O mesmo padrão de crescimento foi observado anteriormente para S. alterniflora no estuário da Lagoa dos Patos (Copertino et al. 1997) e em estuários da América do Norte (Gallagher et al. 1988, Bertness 1991, Tyler \& Zieman 1999), bem como para S. anglica (Thompson et al. 1991) e S. maritima (Castillo et al. 2005). Apesar de algumas evidências de diferenças genotípicas entre populações de Spartina crescendo em distintos níveis da zona entremarés (Gallagher et al. 1988, Proffit et al. 2003), transplantes recíprocos de plantas entre populações de diferentes pisos têm demonstrado reversões das diferenças de tamanho nos colmos (Gallagher et al. 1988, Thompson et al. 1990, Bertness 1991, Proffit et al. 2003, Castillo et al. 2005) e, consequentemente, um forte controle edáfico e do estresse de alagamento sobre esta característica das plantas. Potenciais redox negativos em superfície e nos primeiros decímetros de profundidade, como os observados nas zonas pioneiras, têm sido encontrados em áreas baixas de marismas frequentemente alagadas (Bertness 1991, Tyler \& Zieman 1999, Nieva et al. 2001, Castillo et al. 2005) e relacionados ao acúmulo de sulfetos e outros compostos tóxicos às plantas de marismas, bem como diferenças na disponibilidade de nutrientes entre estas áreas baixas e partes mais altas na zona entremarés (Bradley \& Morris 1990, Tyler \& Zieman 1999, Mendonça \& Costa 2008). Castillo et al. (2005) em seu estudo com 34 populações de $S$. maritima do sudoeste da Espanha, observaram que populações de áreas com baixo potencial redox tenderam a ter maiores alturas de colmos. Segundo estes autores, condições hipóxicas parecem estimular o desenvolvimento dos colmos, o que resultaria em uma oxigenação mais eficiente do substrato por colmos com sistemas aerenquimáticos mais desenvolvidos.

Spartina alterniflora e $S$. densiflora demonstraram diferentes padrões de ocupação espacial das marismas por suas populações pioneiras e maduras. S. alterniflora apresenta um adensamento de colmos na sua população madura em relação à pioneira, enquanto $S$. densiflora reduz grandemente o número de colmos em populações maduras no sudoeste do Atlântico. Estas diferenças podem ser 

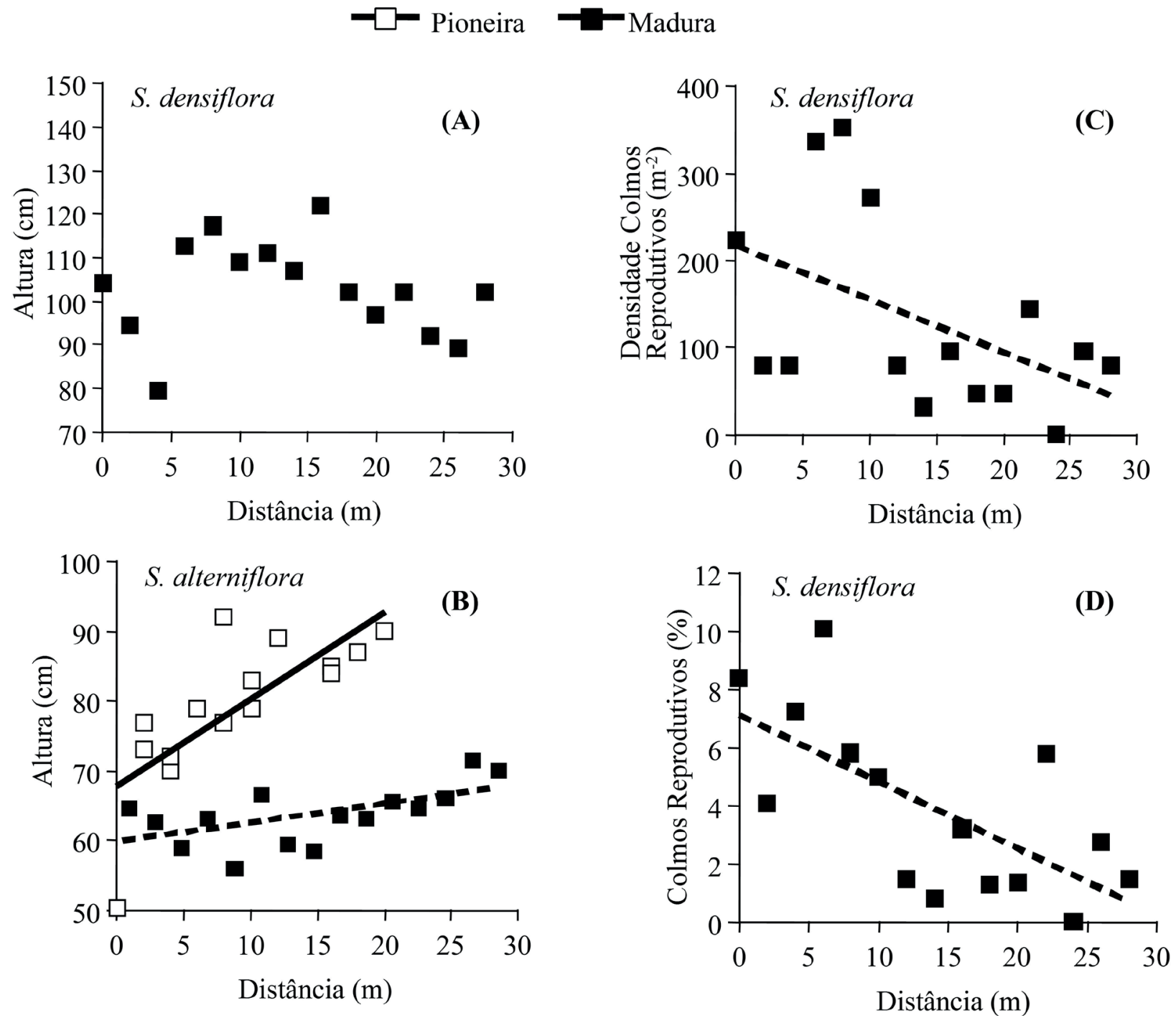

Figs.4 A-D. Variação em relação à distância da linha d'água (planos de lama adjacentes) na altura dos colmos (A, B), densidade (C) e percentagem de colmos reprodutivos (D) de populações pioneiras e maduras de S. alterniflora (Sa) e S. densiflora (Sd) em marismas da Ilha da Pólvora (Rio Grande, RS), que apresentaram alta variabilidade intrapopulacional. Linhas de regressões lineares são apresentadas quando um efeito significativo $(\mathrm{p}<0,05)$ da distância na variável foi detectado.

explicadas pelos diferentes hábitos rizomatosos. A formação espaçada de colmos de S. alterniflora, devido grandes distâncias entrenós do rizoma (forma de crescimento "guerrilla" sensu Lovett Doust \& Lovett Doust 1982), resulta em baixa densidade de colmos nas áreas pioneiras. Com o passar do tempo, ramificações dos rizomas, brotamento de colmos e a germinação de sementes, novos colmos ocupam o espaço disponível, adensando a marisma. Semelhantemente, Dai \& Wiegert (1996) encontraram uma densidade média de colmos de $S$. alterniflora cinco vezes menor em uma marisma jovem do que em uma marisma madura na Georgia (E.U.A.). Plantas de Spartina densiflora, com pequena distância entrenós nos rizomas, investe pesadamente na formação de colmos (formando tufos) para colonização de novos hábitats (forma de crescimento "phalanx" sensu Lovett Doust \& Lovett Doust 1982). As densidades da população pioneira de S. densiflora (2.00012.000 colmos $\mathrm{m}^{-2}$ ) foram semelhantes às citadas para esta espécie por outros autores em outras localidades da América do Sul (Vicari 2002, Martinetto et al. 2016) e da Espanha (Nieva et al. 2005). Esta forma de crescimento de $S$. densiflora é altamente competitiva, por dificultar a ocupação das marismas jovens por outras espécies, promovendo escassez de luz e espaço aéreo (Castellanos et al. 1988, Costa et al. 2003), além de favorecer a retenção de partículas e maior resistência mecânica ao fluxo das marés (Nieva et al. 2001). Da mesma forma, a redução da densidade de colmos em populações maduras de $S$. densiflora também foi observada por Nieva et al. (2005) em marismas no sudoeste da Espanha. Esta redução em marismas maduras, mais elevadas no entremarés, parece estar relacionada com mortalidade dos colmos após frutificação (Nieva et al. 2001), visto que os colmos de Spartina são monocárpicos (Castellanos et al. 1998; Nieva et al. 2001). Esta mortalidade, aparentemente, não é acompanhada por uma reposição na mesma magnitude, 
pela formação de novos rizomas com botões germinativos para darem origem a novos colmos.

Houve uma marcada redução no investimento reprodutivo entre marismas pioneiras e maduras para ambas as espécies. Neste estudo, 14\% dos colmos da população pioneira de $S$. alterniflora floresceram, enquanto a população madura com mais de 50 anos não floresceu. Estudos anteriores de $S$. alterniflora no estuário Lagoa dos Patos também registraram altos valores de proporção de colmos reprodutivos (40-70\%) em populações pioneiras desta espécie (Copertino et al. 1997, Costa \& Marangoni 1997, Mendonça \& Costa 2008, Marangoni \& Costa 2012). De forma semelhante, Castellanos et al. (1998) observaram ausência de floração em população madura de S. maritima e uma incidência de $17 \%$ de floração em população pioneira da mesma espécie. Segundo estes autores a redução do florescimento em populações maduras pode estar relacionada a um mecanismo densidade-dependente da floração, experimentalmente demonstrado por Thompson et al. (1990) pela adição de nutrientes em populações maduras de $S$. anglica. O aumento da disponibilidade de nutrientes por colmo pode ser um importante determinante do investimento reprodutivo. Tyler \& Zieman (1999) observaram que as marismas mais ricas em nutrientes (fosfato e amônio) apresentavam maiores porcentagens de colmos reprodutivos de S. alterniflora (24-35\%) do que marismas pobres em nutrientes (13-15\%) na costa Atlântica norte-americana.

Diferente das demais espécies de Spartina citadas acima, a proporção de colmos reprodutivos de $S$. densiflora variou pouco entre populações pioneira e madura (médias globais entre 4 a $6 \%$ ), entretanto o número absoluto de inflorescências produzidas pela população pioneira foi quatro vezes superior à madura, devido a maior densidade total de colmos nesta primeira população. Contrastantemente, Nieva et al. (2001) observaram em marismas espanhóis diferenças marcantes na proporção de colmos reprodutivos de populações pioneiras (5 a 37\%) e maduras de $S$. densiflora $(0,07$ a $0,27 \%)$. Um baixo investimento reprodutivo também foi observado por Vicari et al. (2002) para uma população madura de $S$. densiflora na Argentina; $1,4 \%$ de colmos reprodutivos. As médias globais de porcentagens de colmos reprodutivos de $S$. densiflora encontradas em nosso estudo foram baixas, inclusive quando comparadas com os $24 \%$ descritos por Costa \& Marangoni (1997) em uma população pioneira na mesma ilha.

A fecundidade da população pioneira de S. alterniflora da Ilha da Pólvora foi extremamente baixa, produzindo em média apenas 0,4 cariopses por inflorescência ou 76 cariopses $\mathrm{m}^{-2}$. Este resultado é semelhante ao de populações invasoras de $S$. alterniflora na Baia de San Francisco (E.U.A.), que produziram de 10 a 30 espiguetas por racemo (Daehler 1998) ou entre 250 e 660 espiguetas por inflorescência (considerando até 25 racemos por inflorescência; Mobberley 1956), mas apenas 0,8 cariopses por inflorescência (Callaway \& Josselyn 1992). Entretanto, a produção de cariopses por $S$. alterniflora é irregular, com uma grande variabilidade interanual e local (Daehler \& Strong 1994). Intensificação de fatores inibitórios dos processos de polinização anemófila e de fecundação cruzada (característicos de S. alterniflora; Fang et al. 2004), como chuvas excessivas no período de polinização, poderiam explicar o grande número de estruturas florais que não geram cariopses. Esta variação na produção de cariopses pode estar também relacionada à predação de inflorescências por insetos e aves (Bertness \& Shumway 1992), bem como variações nas populações de polinizadores e ocorrência de processos abortivos como mecanismo de defesa contra a autofecundação (Daehler \& Strong 1994). A ação de predadores de sementes e a contaminação por fungo (Claviceps) foram observadas em muitas espiguetas, mas não quantificados neste estudo e poderiam ter atacado frutos em formação, prejudicando o desenvolvimento das cariopses.

As populações de $S$. densiflora demonstraram maior eficiência média na produção de cariopses (20 cariopses de 94 espiguetas por inflorescência; população madura) e maior fecundidade (16.258 cariopses $\mathrm{m}^{-2}$; população pioneira) do que populações de S. alterniflora. Uma maior produção de cariopses por $S$. densiflora promove um mecanismo eficiente de dispersão e colonização de novas áreas (Callaway \& Josselyn 1992, Daehler \& Strong 1994, Nieva et al. 2001). Devido à pequena capacidade de extensão horizontal dos rizomas de $S$. densiflora (Costa \& Marangoni 1997, Marangoni \& Costa 2012), sua ocupação espacial ocorre principalmente pela grande produção e dispersão de sementes, cuja germinação permite a formação de vários núcleos de colonização vegetativa de planos de lama. A combinação da saturação do ambiente com sementes e a alta densidade de colmos das populações pioneiras é um efetivo mecanismo de ocupação de áreas não vegetadas e de exclusão de espécies nativas de marismas invadidas por S. densiflora na Europa (Nieva et al. 2001) e Costa Pacífica da Califórnia (Kittelson \& Boyd 1997).

Apesar das marcadas diferenças entre populações pioneiras e maduras, mais de $50 \%$ da variabilidade nas alturas das plantas de ambas as espécies foi explicada por diferenças intrapopulacionais. No caso específico de $S$. alterniflora esta variabilidade local pode ser relacionada por um estímulo ao crescimento em altura com o afastamento da linha d'água-borda do plano de lama. Proffit et al. (2003) descreveram maiores alturas de plantas de $S$. alterniflora nos centros do que nas bordas de tufos crescendo por dois anos em planos de lama, atribuindo esta resposta a maximização da captura dos recursos. Segundo estes autores, melhores capturas de espaço e recursos no sedimento ainda não ocupado são alcançadas com uma forma de crescimento baseada em vários pequenos colmos (mais perfilhação), em vez de poucos colmos de grande porte. Dados de adição de fosfato e amônio em plantios na zona entremarés (Mendonça \& Costa 2008) e em condições controladas 
de casa de vegetação (Azevedo et al. 2016), sugerem que alta disponibilidade destes elementos sob baixas razões $\mathrm{N}$ :P induzem a perfilhação de S. alterniflora e consequente rápida ocupação espacial. Na situação de campo, baixas razões $\mathrm{N}: \mathrm{P}($ i.e.; $2,5: 1)$ ocorrem em solos com baixos valores negativos de redox, que favorecem a liberação de fósforo ligado a hidróxidos e óxidos, bem como a denitrificação (Tyler e Zieman 1999, Costa et al. 2003, Mendonça \& Costa 2008). Quantidades médias significativamente maiores de ortofosfato foram encontradas em planos entremarés não vegetados $\left(68 \mathrm{mg} \mathrm{kg}^{-1}\right)$ do que em marismas jovens de $S$. alterniflora (49-53 $\mathrm{mg} \mathrm{kg}^{-1}$ ) ou no interior de marismas maduras de $S$. densiflora (38 $\left.\mathrm{mg} \mathrm{kg}^{-1}\right)$ (Costa et al. 2003, Marangoni \& Costa 2012). Consequentemente, condições nutricionais estimulantes à perfilhação de S. alterniflora na borda de suas populações com planos não vegetados, parecem ser determinantes do menor desenvolvimento vertical dos seus colmos nestes habitats transicionais.

A variação espacial nas condições ambientais também afeta a densidade e a proporção de colmos reprodutivos de S. densiflora na população madura, cujos valores médios diminuem com o aprofundamento na matriz vegetada. Estes resultados sugerem que, como observado entre marismas pioneiras e maduras, a floração desta espécie é estimulada pela proximidade com áreas mais frequentemente alagadas onde há maior perfilhação. Este padrão espacial pode ser o resultado de pelo menos dois processos. O primeiro processo, mencionado anteriormente, resulta da combinação da monocarpia dos colmos e a pequena formação de novos rizomas com botões germinativos, que possam originar novos colmos em áreas ocupadas por S. densiflora a várias décadas (Nieva et al. 2001). O segundo processo poderia ser a redução da disponibilidade de fósforo, devido à elevação do terreno por ação biogênica de $S$. densiflora, que pode aumentar do potencial redox (Nieva et al. 2001, 2005). A densa rede de raízes com aerênquima promove a formação de ferro oxidado e óxido de alumínio, compostos capazes de fixar o fosfato, tornando esse nutriente menos disponível no sedimento (Baumgarten et al. 2001, Mendonça \& Costa 2008). Contrastantemente, processos que aceleram a degradação/mineralização do detrito na marisma, tais como incêndios ou intensa atividade de herbivoria e/ou escavação por caranguejos da zona entremarés, aumentam a proporção de colmos reprodutivos de populações maduras de S. densiflora (Nieva et al. 2001, Bortolus et al. 2004).

Este estudo demonstrou uma grande variação na altura de colmos, na densidade de colmos e na abundância de estruturas reprodutivas entre diferentes estágios de desenvolvimento de marismas recobertas pelas gramíneas $S$. alterniflora e $S$. densiflora. Populações pioneiras de $S$. densiflora utilizam de alta fecundidade para nucleação, a partir de sementes que resultam em novos tufos de colmos sobre planos de lama não colonizados, enquanto S. alterniflora ocupa o espaço disponível através do alongamento de seus rizomas e perfilhamento de vários colmos com pequena altura. Ambas as espécies mantêm suas populações de marismas maduras principalmente através da propagação vegetativa. Fatores edáficos relacionados com o potencial redox e a disponibilidade de nutrientes parecerem ser determinantes de alta variabilidade na altura (ambas as espécies) e na abundância de estruturas reprodutivas (S. densiflora) dentro de populações pioneiras e maduras.

\section{AGRADECIMENTOS}

As bolsas de doutorado de Vera Lúcia Caetano e Adriana Martins Guedes de Azevedo foram concedidas pela Coordenação de Aperfeiçoamento de Pessoal de Nível Superior. O Conselho Nacional de Desenvolvimento Científico e Tecnológico apoiou este projeto através da bolsa de pesquisa de César Serra Bonifácio Costa e da bolsa de mestrado CT-Hidro de Gabriela Greis.

\section{REFERÊNCIAS}

Azevedo, A.M.G., Costa, C.S.B., Leal, C.E.S. \& Marangoni, J.C. 2016. Efeitos da densidade de plantio e da adição de nutrientes na produção de mudas de gramas halófitas em recipientes. Revista Ceres 63:76-85.

Baumgarten, M.G.Z., Niencheski, L.F.H. \& Veeck, L. 2001. Nutrientes na coluna da água e na água intersticial de sedimentos de uma enseada rasa estuarina com aportes de origem antrópica (RS- Brasil). Atlântica 23:101-116.

Bertness, M.D. 1991. Zonation of Spartina patens and Spartina alterniflora in a New England salt marsh. Ecology 72(1):138-148.

Bertness, M.D. \& Shumway, S.W. 1992. Consumer driven pollen limitation of seed production in marsh grasses. American Journal of Botany 79:288-293.

Bortolus, A., Laterra, P. \& Iribarne, O.O. 2004. Crab-mediated phenotypic changes in Spartina densiflora Brong. Estuarine, Coastal and Shelf Science 59:97-107.

Bradley, P.M. \& Morris, J.T. 1990. Physical characteristics of salt marsh sediments: ecological implications. Marine Ecology Progress Series 61:245-252.

Callaway, J.C. \& Josselyn, M.N. 1992. The introduction and spread of smooth cordgrass (Spartina alterniflora) in south San Francisco Bay. Estuaries 15(2):218-226.

Castellanos, E.M., Heredia, C., Figueroa, M.E. \& Davy, A.J. 1998. Tiller dynamics of Spartina maritima in successional and non-successional Mediterranean salt marsh. Plant Ecology 137:213-225.

Castillo, J.M., Redondo, S., Wharmby, C., Figueroa, M.E., Castellanos, E.M. \& Davy, A.J. 2005. Environmental determination of shoot height in populations of the cordgrass Spartina maritima. Estuaries 28(5):761-766.

Chung, C. 2006. Forty years of ecological engineering with Spartina plantations in China. Ecological Engineering 27: 49-57.

Copertino, M., Costa, C.S.B. \& Seeliger, U. 1997. Dinâmica populacional de Spartina alterniflora em pântanos salgados do estuário da Lagoa dos Patos, Rio Grande, RS. In Anais do VIII Seminário Regional de Ecologia. Universidade Federal de São Carlos, São Carlos, p. 295-312.

Costa, C.S.B. 1998. Plantas de marismas e terras alagáveis. In Os ecossistemas costeiro e marinho do extremo Sul do Brasil (U. Seeliger, C. Odebrecht \& J.P. Castello, eds.). Editora Ecoscientia, Rio Grande, p. 25-29.

Costa, C.S.B. \& Davy, A.J. 1992. Coastal saltmarsh communities of Latin America. In Coastal plants community of Latin America (U. Seeliger, ed.). Academic Press, New York, p.179-199.

Costa, C.S.B., Iribarne, O.O. \& Farina, J.M. 2009. Human impacts and threats to the conservation of South American salt marshes. In Salt marshes under global siege (B.R. Silliman, T. Grosholtz \& M.D. Bertness, eds.). University of California Press, Berkeley, p. 337-359.

Costa, C.S.B. \& Marangoni, J.C. 1997. Criação de marismas com espécies nativas: experimento piloto no sul do Brasil. In Resumos 
Expandidos do VII congresso Latino Americano de Ciências do Mar. Instituto Oceanográfico da Universidade de São Paulo, Associacíon Latinoamericana de Investigadores en Ciências del Mar - Federação das Indústrias de São Paulo, Santos, p. 200-201.

Costa, C.S.B.; Marangoni, J.C. \& Azevedo, A.M.G. 2003. Plant zonation in irregularly flooded salt marshes: relative importance of stress tolerance and biological interactions. Journal of Ecology 91(6):951965.

Costa, C.S.B., Seeliger, U., Oliveira, C.P.L. \& Mazo, A.M. 1997. Distribuição, funções e valores das marismas e pradarias submersas no estuário da Lagoa dos Patos (RS, Brasil). Atlântica, Rio Grande 19:67-85.

Cunha, S.R., Asmus, M. \& Costa, C.S.B. 2005. Production dynamics of Spartina alterniflora salt marshes in the estuary of Patos Lagoon (RS, Brazil): A Simulation model approach. Brazilian Journal of Aquatic Science and Technology 9(2):75-85.

Daehler, C.C. 1998. Variation in self-fertility and reproductive advantage of self-fertility for an invading plant (Spartina alterniflora). Evolutionary Ecology 12:553-568.

Daehler, C.C. \& Strong, D.S. 1994. Variable Reproductive output among clones of Spartina alterniflora (Poaceae) invading San Francisco Bay, California: the influence of herbivory, polination, and establishment site. American Jounal of Botany 81(3):307-313.

Dai, T. \& Wiegert, R.G. 1996. Ramet populations dynamics and net aerial primary productivity of Spartina alterniflora. Ecology 77(1):276-288.

Fang, X., Subudhi, P.K., Bradley, C.V. \& Harrison, S.A. 2004. Mode of pollination, pollen germination, and seed in smooth cordgrass (Spartina alterniflora, Poaceae). International Journal Plant Science 165(3):395-401.

Gallagher, J.L., Somers, G.F., Grant, D.M. \& Seliskar, D.M. 1988. Persistent differences in two forms of Spartina alterniflora: a common garden experiment. Ecology 69(4):1005-1008.

Giacobbo, E.O. \& Boechat, S.C. 1988. O gênero Spartina Schreber (Gramineae, Chloridoideae) no Rio Grande do Sul. Iheringia. Série Botânica 37:89-109.

Hulzen, J.B., Van Soelen, J. \& Bouma, T.J. 2007. Morphological variation and habitat modification are strongly correlated for the autogenic ecosystem engineer Spartina anglica (common cordgrass). Estuaries and Coasts 30(1):3-11.

Kittelson, P.M. \& Boyd, M.J. 1997. Mechanisms of expansion for an introduced species of cordgrass, Spartina densiflora, in Humboldt Bay, California. Estuaries 20:770-778.

Linthurst, R.A. \& Seneca, E.D. 1981. Aeration, nitrogen and salinity as determinants of Spartina alterniflora Loisel. growth response. Estuaries 4(1):53-63.

Lovett Doust, L. \& Lovett Doust, J. 1982. The battle strategies of plants. New Scientist 8:81-84.
Marangoni, J.C. \& Costa, C.S.B. 2012. Short-and long-term vegetative propagation of two Spartina species on a salt marsh in Southern Brazil. Estuaries and Coasts 35:763-773.

Martinetto, P., Montemayor, D.I., Alberti, J., Costa, C.S.B. \& Iribarne, O.O. 2016. Crab bioturbation and herbivory may account for variability in carbon sequestration and stocks in south west Atlantic salt marshes. Frontiers in Marine Science 3:122.

Mobberley, D.G. 1956. Taxonomy and distribution of the genus Spartina. Iowa State College Journal of Science 30(4):471-574.

Mendonça, P.T. \& Costa, C.S.B. 2008. Efeitos da disponibilidade de nitrogênio e fósforo no estabelecimento de Spartina alterniflora em um plano entremarés irregularmente alagado. Neotropical Biology and Conservation 3(3):135-148.

Nieva, F.J., Castellanos, E.M., Castillo, J.M. \& Figueroa, M.E. 2005. Clonal growth and tiller demography of the invader cordgrass Spartina densiflora Brongn. at two contrasting habitats in SW European salt marshes. Wetlands 25(1):122-129.

Nieva, F.J., Díaz-Espejo, A., Castellanos, E.M. \& Figueroa, M.E. 2001. Field variability of invanding populations of Spartina densiflora Brongn. in differents habitats of the Odiel Marshes (SW Spain). Estuarine, Coastal and Shelf Science 52:515-527.

Peixoto, A. \& Costa, C.S.B. 2004. Produção primária líquida aérea de Spartina densiflora Brong. (Poaceae) no estuário da laguna dos Patos, Rio Grande do Sul, Brasil. Iheringia. Série Botânica 59(1):27-34.

Proffit, C.E., Travis, S.E. \& Edwards, K.R. 2003. Genotype and elevation influence Spartina alterniflora colonization and growth in created salt marsh. Ecological Applications 13:180-192.

Seliskar, D.M., Gallagher, J.L., Burdick, D.M. \& Mutz, L.A. 2002. The regulation of ecosystem functions by ecotypic variation in the dominant plant: a Spartina alterniflora salt-marsh case study. Journal of Ecology 90:1-11.

Thompson, J.D., Gray, A.J. \& McNeilly, T. 1990. The effects of density on the population dynamics of Spartina anglica. Acta Oecologica 11(5):669-682.

Thompson, J.D., McNeilly, T. \& Gray, A.J. 1991. Population variation in Spartina anglica: Evidence from a common garden experiment. New Phytologist 117:115-128.

Tyler, A.C. \& Zieman, J.C. 1999. Patterns of development in the creekbank region of a barrier island Spartina alterniflora marsh. Marine Ecology Progress Series 180:161-177.

Vicari, R.L.F., Madanes, N., Bonaventura, S.M. \& Pancotto, V. 2002. Tiller population dynamics and production on Spartina densiflora (Brong.) on the floodplain of the Paraná river, Argentina. Wetlands 22(2):347-354.

Vieira, E.F. 1983. Rio Grande: geografia física, humana e econômica. Sagra, Porto Alegre. 158p.

Zar, J.H. 2010. Biostatistical Analysis. Prentice Hall, Englewood Cliffs. 944p. 